\title{
Nuevas aportaciones a la flora vascular en el norte de la península ibérica.
}

\section{New contributions to the vascular flora in the north of the Iberian Peninsula.}

Alfred Llorente-Rodrigo ${ }^{1}$, José Antonio Cadiñanos-Aguirre ${ }^{2}$

\section{Resumen}

Se citan varios taxones interesantes de la flora vascular del norte de la península ibérica, concretamente de las provincias vascas de Araba/Álava, Bizkaia y Gipuzkoa y de las comunidades de Navarra, Cantabria, Asturias, La Rioja y Castilla-León.

Se mencionan 6 primeras citas provinciales, 17 taxones de flora amenazada o protegida, 6 especies orófitas que presentan cierto efecto pedinótico y 12 especies alóctonas en los citados territorios. También se indican 2 primeras citas para la margen derecha vizcaína y 3 primeras citas para la franja oriental cántabra, además de otras citas provinciales relevantes por su rareza.

Asimismo, se incluyen algunas especies más comunes cuyo interés radica en determinados aspectos biogeográficos (e.g. rebajan la altitud mínima conocida, dan continuidad geográfica en zonas donde no estaban citadas, se presentan en un hábitat peculiar no habitual).

Palabras clave: Spermatophyta, corología, flora amenazada, norte península ibérica, País Vasco, Navarra, Cantabria, Asturias, La Rioja, Castilla y León, primeras, segundas y terceras citas, efecto pedinótico, especies alóctonas, aspectos biogeográficos.

\footnotetext{
1 Sociedad Micológica y Botánica de Getxo "Basozaleak" y socio de Fotografía y Biodiversidad (FyB); https://www. biodiversidadvirtual.org/.

* Correspondencia: Ilorentealfred@outlook.es

2 Departamento de Geografía, Prehistoria y Arqueología, UPV/EHU Sociedad de Ciencias Aranzadi; joseacadi@hotmail.com.

* Correspondencia: joseacadi@hotmail.com
} 


\begin{abstract}
Several interesting taxa of vascular flora of the North of the Iberian Peninsula are recorded, specifically in the Basque territories of Araba/Álava, Bizkaia and Gipuzkoa and in the regional communities of Navarra, Cantabria, Asturias, La Rioja and Castile and Leon.

Six first provincial records are mentioned, 17 taxa of threatened or protected flora, 6 orophytic species that have a certain pedinotic effect and 12 non-native species in the afore-mentioned territories. There are also 2 first records for eastern Bizkaia and 3 first records for the eastern fringe of Cantabria, in addition to other relevant provincial records due to their rarity.

Likewise, a number of more common species whose interest lies in certain biogeographical aspects are included (e.g. they lower the known minimum altitude, they provide geographical continuity in areas where they were not mentioned or they occur in a peculiar and unusual habitat).
\end{abstract}

Key words: Spermatophyta, chorology, threatened flora, northern Iberian Peninsula, Basque Country, Navarra, Cantabria, Asturias, La Rioja, Castile and Leon. First, second and third records, pedinotic effect, non-native species, biogeographical aspects.

\title{
Laburpena
}

Iberiar penintsulako iparraldeko landare baskularren taxon interesgarri batzuk aipatzen dira, Araba, Bizkaia eta Gipuzkoa herrialdeetan, baita Nafarroa, Kantabria, Asturias, Errioxa eta Gaztela-Leoneko erkidegokoetan ere.

Lehenbiziko probintzia mailako 6 aipu dira, mehatxatutako edo babestutako floraren 17 taxon, neurri batean efektu pedinotikoa erakusten duten 6 espezie orofitiko eta aztertutako lurraldeetan 12 espezie bertakotuak. Bizkaiko Eskuinaldeko 2 lehen aipamen eta Kantabriako ekialdeko 3 lehen aipu ere badaude; baita urritasunagatik garrantzitusak diren beste probintzia batzuetako aipamen batzuk ere.

Era berean, zenbait alderdi biogeografikotan interesa duten espezie arruntago batzuk sartu dira (e.g. ezagutzen den gutxieneko altuera jaisten dutenak; aipaturik ez zeuden guneetan segida geografikoa ematen dutenak; habitat berezi eta ezohiko batzuetan agertzen direnak).

Gako hitzak: Spermatophyta, korologia, flora mehatxatua, iberiar penintsulakko iparraldea, Euskadi, Nafarroa, Kantabria, Asturias, Errioxa, Gaztela eta Leon., lehen, bigarren eta hirugarren aipamen, efektu pedinotiko, bertakotutako espezieak, alderdi biogeografikoak.http://www.aranzadi.eus/ linkean.

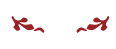


Como en artículos anteriores, el presente es el resultado de diversos itinerarios botánicos por el norte peninsular, en concreto por las siguientes provincias o comunidades autónomas: Araba/Álava, Asturias, Burgos, Cantabria, Gipuzkoa, La Rioja, Navarra y Bizkaia. En esta ocasión, incorporamos citas sobre las que, con anterioridad, teníamos dudas acerca de la idoneidad de su divulgación, pero, visto el abundante caudal de información que sobre este particular está apareciendo en los últimos años, hemos optado por darlas a conocer.

El objetivo principal de este trabajo es ampliar el conocimiento de las plantas y su distribución en nuestro territorio, prestando especial atención a aquellas que suponen una novedad provincial o regional, las que están incluidas en los diferentes catálogos de flora protegida, las plantas montanas acomodadas a vivir en zonas bajas (i.e. pedinosis, Font Quer, 1993: 811) o las alóctonas cuya presencia era desconocida o se están extendiendo en nuestra zona habitual de trabajo. Asimismo, hemos añadido aquellas especies cuyo interés radica en aspectos biogeográficos como la cota altitudinal (o, simplemente, la altitud), un nexo geográfico regional o interprovincial, o un hábitat peculiar poco habitual, entre otros.

El orden en la relación de los taxones y, en cada uno de ellos, de las abreviaturas de las provincias (según Flora Iberica) es alfabético. En cada provincia la disposición de las citas es cronológica. Aunque no se mencionen expresamente en las citas, se conservan los pliegos de las plantas en los herbarios particulares de los autores (Alfred Llorente: ALF, J. A. Cadiñanos: Arangu-Angi-), salvo de aquellas plantas de determinación razonablemente segura o cuya recolección, a nuestro juicio, podría suponer un riesgo para la pervivencia de la nueva población encontrada. En tal caso, hemos sustituido el material de herbario por fotografías tomadas en el propio lugar del hallazgo o en estudio, de manera que, de cada cita disponemos de material herborizado o soporte gráfico y, a veces, de ambas evidencias. En todos los casos, se anotan las coordenadas UTM (cuadrícula de un $\mathrm{km}^{2}$ ) y se facilitan las referencias ecológicas y geográficas imprescindibles.

Para la nomenclatura nos hemos basado principalmente en Flora Iberica (Castroviejo et al., 1980-2020) tanto de los volúmenes publicados, como de los borradores disponibles en línea. Para la corología, además de consultar también dicha flora, hemos tomado Aizpuru et al. (1999) como referencia principal, dado que el marco de estudio de nuestro trabajo está centrado en el norte peninsular. En Cantabria, el inventario ineludible es la obra de Durán (2014) y adiciones (Durán et al., 2017 y 2019). Para Burgos lo es el libro de Alejandre et al. (2006) con todas sus adiciones posteriores (Alejandre et al., de 2008 a 2017) y, para Navarra, el de Lorda (2013).

Debido a lo extenso del documento original, a sugerencia de la redacción de esta revista, hemos acordado publicar en formato impreso esta versión reducida del artículo, mediante dos tablas sintéticas. La versión extendida con la relación exacta de todas las citas y localidades, los comentarios de cada taxón, las referencias a anteriores citas publicadas, la bibliografía utilizada y diversas fotografías, se puede consultar en 
el siguiente enlace de acceso exclusivo online en la página web de Munibe Ciencias Naturales - Natur Zientziak: http://www.aranzadi.eus/munibe-natur-zientziak.

Se mencionan 138 taxones de plantas vasculares poco frecuentes, raras o muy raras en el norte peninsular y 214 localidades (en la versión extendida hay más citas porque de algunas localidades se aportan más de una cita) de las cuales 80 corresponden a Bizkaia (BI), 59 a Burgos (BU), 57 a Cantabria (S), 9 a Araba/Álava (VI), 5 a Asturias (O), 2 a Navarra (NA) y 1 a Gipuzkoa (SS) y La Rioja (LO); abreviaturas provinciales según Flora Iberica). Entre estos registros de citas, algunas son primeras citas provinciales, como Anemone hortensis, Crocus chrysanthus, Primula x polyantha, Pulsatilla rubra, Scrophularia peregrina y Vicia loiseleurii. Citamos también plantas que están incluidas en los diferentes catálogos de flora amenazada o protegida de las provincias prospectadas, como Arisarum simorrhinum, Campanula adsurgens, Daphne cneorum, Drosera intermedia, Dryopteris aemula, Erodium daucoides, Euphorbia nevadensis subsp. aragonensis, Hypericum caprifolium, Illecebrum verticillatum, Inula helvetica, Ononis ramosissima, Pinguicula lusitanica, Pulsatilla rubra, Sorbus latifolia, Taxus baccata, Vandenboschia speciosa y Woodwardia radicans. Como en nuestros artículos precedentes, siempre prestamos especial atención a aquellas especies orófitas que presentan cierto efecto pedinótico, como Ajuga pyramidalis subsp. pyramidalis, Daphne cneorum, Dryopteris oreades, Eryngium bourgatii, Merendera montana y Thalictrum minus subsp. minus. También queremos destacar la presencia de especies alóctonas en los territorios prospectados, algunas de las cuales parecen proliferar de manera impetuosa, como Amsinckia calycina, Chondrilla juncea, Cotula australis, Crocus chrysanthus, Duchesnea indica, Gamochaeta coarctata, Lathyrus ochrus, Lavatera trimestris, Rhus coriaria, Salpichroa origanifolia, Sisyrinchium angustifolium y Sisyrinchium rosulatum. Por último, hemos corregido algunos nombres de especies publicadas en artículos anteriores: Gamochaeta coarctata y Soliva sessilis.

A continuación, adjuntamos una tabla resumen con los diferentes motivos que han justificado la publicación de las citas y el número de citas por localidad ( $n=243$; algunas citas presentan más de una) que hacen referencia a cada uno de estos motivos.

\begin{tabular}{|lc|}
\hline ¿Porqué se citan? & Número \\
\hline Flora protegida & 22 \\
\hline $1^{\text {a }}$ cita provincial & 5 \\
\hline Efecto pedinótico & 8 \\
\hline Especie alóctona & 21 \\
\hline Correción & 4 \\
\hline $1^{\text {a }}$ cita margen derecha vizcaína & 3 \\
\hline Franja costera vizcaína & 13 \\
\hline
\end{tabular}




\begin{tabular}{|lc|}
\hline ¿Porqué se citan? & Número \\
\hline 1 $^{\text {c cita oriental cántabra }}$ & 5 \\
\hline Franja costera oriental cántabra & 11 \\
\hline $2^{\text {a cita provincial }}$ & 8 \\
\hline $2^{\circ}$ cita mitad septentrional burgalesa & 1 \\
\hline $3^{\text {a cita provincial }}$ & 7 \\
\hline $4^{\text {a cita provincial }}$ & 2 \\
\hline $5^{\text {a cita provincial }}$ & 1 \\
\hline Cota altitudinal & 22 \\
\hline Cota altitudinal (efecto oreinótico) & 1 \\
\hline Rebaja cota altitudinal cántabra & 1 \\
\hline Rebaja cota altitudinal peninsular & 2 \\
\hline Ampliación corológica & 89 \\
\hline Ampliación corológica (nexo geográfico) & 13 \\
\hline Índice de frecuencia & 2 \\
\hline Alejada franja litoral & 1 \\
\hline Población finícola & 1 \\
\hline
\end{tabular}

Y en la siguiente tabla de especies de esta versión impresa y abreviada del manuscrito mostramos únicamente la relación completa de taxones, la provincia (mediante los símbolos usuales en Flora Iberica y publicaciones similares) y localidades de las citas (sólo mencionamos una vez la localidad, aunque hubiera más de una cita en ella) y, sobre todo, el motivo que ha originado su publicación en el presente trabajo, para facilitar la consulta de la versión extendida online a los lectores interesados.

\begin{tabular}{|c|c|c|c|}
\hline Nombre científico & PR & Localidad & ¿Porqué se citan? \\
\hline Aceras anthropophorum & $S$ & Castro Urdiales & $\begin{array}{c}\text { Franja costera oriental } \\
\text { cántabra }\end{array}$ \\
\hline \multirow[b]{2}{*}{ Aegonychon purpurocaeruleum } & $\mathrm{BI}$ & Zalla y Güeñes & Índice de frecuencia \\
\hline & $S$ & Castro Urdiales & $\begin{array}{c}\text { Franja costera } \\
\text { oriental cántabra }\end{array}$ \\
\hline Agrostis hesperica & $S$ & Castro Urdiales y Guriezo & Ampliación corológica \\
\hline Ajuga pyramidalis s. pyramidalis & $S$ & Castro Urdiales y Guriezo & Efecto pedinótico \\
\hline Allium ampeloprasum & $\mathrm{BI}$ & Derio & Ampliación corológica \\
\hline Allium ursinum s. ursinum & $\mathrm{BU}$ & Villasuso de Mena y Herrán & Ampliación corológica \\
\hline Althaea hirsuta & $\mathrm{BI}$ & Mungia y Sopelana & $\begin{array}{l}1^{\text {a }} \text { cita margen } \\
\text { derecha vizcaína }\end{array}$ \\
\hline
\end{tabular}




\begin{tabular}{|c|c|c|c|}
\hline Nombre científico & PR & Localidad & ¿Porqué se citan? \\
\hline Amsinckia calycina & $\mathrm{BU}$ & Villacomparada de Rueda & $\begin{array}{l}\text { Especie alóctona / } \\
2^{\text {a cita burgalesa }}\end{array}$ \\
\hline Anemone hortensis & BU & Extramiana & $1^{\text {a }}$ cita provincial \\
\hline Angelica major & $\mathrm{BI}$ & Balmaseda & Ampliación corológica \\
\hline \multirow[t]{2}{*}{ Aphanes arvensis } & $S$ & Castro Urdiales & Ampliación corológica \\
\hline & $\mathrm{BI}$ & Sopuerta & Ampliación corológica \\
\hline Arisarum simorrhinum & $S$ & Castro Urdiales & Flora protegida \\
\hline Aster alpinus & 0 & Cangas de Onís y Parres & Ampliación corológica \\
\hline Atriplex patula & $\mathrm{Bl}$ & Axpe-San Bartolomé & Franja costera vizcaína \\
\hline \multirow[t]{2}{*}{ Barbarea vulgaris } & $\mathrm{BU}$ & La Puebla de Arganzón & Ampliación corológica \\
\hline & $\mathrm{VI}$ & Maturana & Ampliación corológica \\
\hline Bolboschoenus maritimus & $\mathrm{BU}$ & Medina de Pomar & Ampliación corológica \\
\hline Brachypodium distachyon & $S$ & Castro Urdiales & $1^{\text {a }}$ cita oriental cántabra \\
\hline Calamagrostis arundinacea & $S$ & Castro Urdiales & Ampliación corológica \\
\hline Campanula adsurgens & 0 & Somiedo & Flora protegida \\
\hline Carduus nigrescens s. nigrescens & $S$ & Cervatos & $2^{a}$ cita provincial \\
\hline Carduus nutans & $\mathrm{BI}$ & Areatza & Ampliación corológica \\
\hline \multirow[t]{2}{*}{ Caucalis platycarpos } & $\mathrm{VI}$ & Campezo & Ampliación corológica \\
\hline & $\mathrm{BU}$ & Espinosa de Cervera & Ampliación corológica \\
\hline Cephalanthera rubra & $\mathrm{BI}$ & Gorliz & $\begin{array}{l}2^{\text {a }} \text { cita provincial / } \\
\text { Franja costera vizcaína }\end{array}$ \\
\hline Chondrilla juncea & $\mathrm{BI}$ & Leioa & Especie alóctona \\
\hline Colchicum gr. autumnale & $\mathrm{BU}$ & Herbosa y Ribota de Ordunte & Ampliación corológica \\
\hline \multirow[t]{2}{*}{ Cotula australis } & $\mathrm{BI}$ & Cruces y Algorta & Especie alóctona \\
\hline & $S$ & Castro Urdiales & $\begin{array}{l}\text { Especie alóctona/ } \\
1^{\mathrm{a}} \text { cita oriental cántabra }\end{array}$ \\
\hline Crocus chrysanthus & $\mathrm{Bl}$ & Erandio & $\begin{array}{l}1^{\text {a }} \text { cita provincial / } \\
\text { Especie alóctona }\end{array}$ \\
\hline Cucubalus baccifer & BU & Bárcena de Pienza & Ampliación corológica \\
\hline Dactylorhiza insularis & $\mathrm{BU}$ & Espinosa de los Monteros & $\begin{array}{l}\text { Cota altitudinal } \\
\text { (efecto oreinótico) }\end{array}$ \\
\hline Daphne cneorum & $S$ & Guriezo & $\begin{array}{l}\text { Flora protegida / } \\
\text { Efecto pedinótico }\end{array}$ \\
\hline Delphinium gracile & $\mathrm{BU}$ & Pesadas de Burgos & $5^{\text {a }}$ cita provincial \\
\hline Dianthus carthusianorum s. carth. & $\mathrm{BU}$ & Pesadas de Burgos & $\begin{array}{l}\text { Ampliación corológica } \\
\text { (nexo geográfico) }\end{array}$ \\
\hline \multirow{2}{*}{$\begin{array}{l}\text { Doronicum carpetanum s. } \\
\text { pubescens }\end{array}$} & $S$ & Castro Urdiales & Ampliación corológica \\
\hline & $\mathrm{Bl}$ & Balmaseda & Ampliación corológica \\
\hline
\end{tabular}




\begin{tabular}{|c|c|c|c|}
\hline Nombre científico & PR & Localidad & ¿Porqué se citan? \\
\hline Drosera intermedia & S & Castro Urdiales & $\begin{array}{l}\text { Flora protegida / } \\
1^{\circ} \text { cita oriental cántabra }\end{array}$ \\
\hline Dryopteris aemula & $\mathrm{BI}$ & Gatika & $\begin{array}{l}\text { Flora protegida / } \\
\text { Cota altitudinal }\end{array}$ \\
\hline Dryopteris oreades & $S$ & Castro Urdiales & Efecto pedinótico \\
\hline Duchesnea indica & $\mathrm{BI}$ & Berango, Getxo y Derio & Especie alóctona \\
\hline Erica arborea & $S$ & Guriezo & Cota altitudinal \\
\hline Erodium daucoides & BU & Pesadas de Burgos & Flora protegida \\
\hline \multirow{2}{*}{ Eryngium bourgatii } & $S$ & Castro Urdiales & $\begin{array}{l}\text { Efecto pedinótico / } \\
2^{\text {a cita provincial }}\end{array}$ \\
\hline & $\mathrm{BI}$ & Gorliz & $\begin{array}{l}\text { Efecto pedinótico / } \\
2^{\mathrm{a}} \text { cita provincial }\end{array}$ \\
\hline Euphorbia flavicoma s. occidentalis & $S$ & Castro Urdiales & Ampliación corológica \\
\hline $\begin{array}{l}\text { Euphorbia nevadensis s. } \\
\text { aragonensis }\end{array}$ & $\mathrm{BU}$ & $\begin{array}{l}\text { Miranda de Ebro, Ircio y } \\
\text { Hozabejas }\end{array}$ & Flora protegida \\
\hline $\begin{array}{l}\text { Festuca heterophylla s. } \\
\text { heterophylla }\end{array}$ & S & Castro Urdiales y Guriezo & $\begin{array}{l}\text { Ampliación corológica / } \\
\text { Cota altitudinal }\end{array}$ \\
\hline \multirow{2}{*}{ Gamochaeta coarctata } & $\mathrm{BI}$ & $\begin{array}{l}\text { Derio, Sondica, Ranero y } \\
\text { Paules }\end{array}$ & $\begin{array}{l}\text { Especie alóctona / } \\
\text { Corrección }\end{array}$ \\
\hline & BU & Ribota de Ordunte & $\begin{array}{l}\text { Especie alóctona / } \\
3^{\text {a }} \text { cita provincia }\end{array}$ \\
\hline \multirow[t]{2}{*}{ Geranium sanguineum } & $\mathrm{BI}$ & Gorliz & Ampliación corológica \\
\hline & $S$ & Castro Urdiales & Ampliación corológica \\
\hline \multirow[t]{2}{*}{ Hypericum caprifolium } & $\mathrm{VI}$ & Tobillas & Ampliación corológica \\
\hline & $\mathrm{BU}$ & Pedrosa de Tobalina & Flora protegida \\
\hline Hypericum hirsutum & SS & Derio & $3^{\text {a }}$ cita provincial \\
\hline Hypericum tomentosum & $\mathrm{BU}$ & Santa Coloma & $\begin{array}{l}\text { Ampliación corológica } \\
\text { (nexo geográfico) }\end{array}$ \\
\hline Illecebrum verticillatum & SS & Oiartzun & $\begin{array}{l}\text { Flora protegida / } \\
3^{\text {a }} \text { cita provincial }\end{array}$ \\
\hline Inula helvetica & $\mathrm{BU}$ & $\begin{array}{l}\text { Medina de Pomar y } \\
\text { San Millán de San Zadornil }\end{array}$ & Flora protegida \\
\hline Isolepis fluitans & S & Castro Urdiales & Ampliación corológica \\
\hline Lastrea limbosperma & $S$ & Castro Urdiales & Ampliación corológica \\
\hline Lathyrus angulatus & $\mathrm{BU}$ & Basconcillos del Tozo & Ampliación corológica \\
\hline Lathyrus ochrus & $\mathrm{BI}$ & Derio & (nexo geográfico) \\
\hline Lavatera trimestris & $\mathrm{BI}$ & Zamudio y Derio & Especie alóctona \\
\hline Leucanthemum maximum & $\mathrm{BI}$ & Muxika & Especie alóctona \\
\hline \multirow[t]{2}{*}{ Lilium martagon } & $\mathrm{BI}$ & Balmaseda & Ampliación corológica \\
\hline & S & Soba & Ampliación corológica \\
\hline
\end{tabular}




\begin{tabular}{|c|c|c|c|}
\hline Nombre científico & PR & Localidad & ¿Porqué se citan? \\
\hline Lilium pyrenaicum & $\mathrm{BI}$ & Balmaseda & Ampliación corológica \\
\hline Linaria alpina s. filicaulis & $\mathrm{O}$ & Ribadesella & Ampliación corológica \\
\hline Lolium rigidum s. rigidum & $\mathrm{BI}$ & Sopelana & Cota altitudinal \\
\hline Lomelosia stellata & $\mathrm{VI}$ & Apodaka & $\begin{array}{l}\text { Ampliación corológica / } \\
\text { Franja costera vizcaína }\end{array}$ \\
\hline Lythrum portula & $S$ & Castro Urdiales & Ampliación corológica \\
\hline Malus sylvestris & $\mathrm{S}$ & Castro Urdiales y Guriezo & Ampliación corológica \\
\hline Malva trifida & NA & Viana & Ampliación corológica \\
\hline Merendera montana & 0 & Villaviciosa & Efecto pedinótico \\
\hline \multirow{3}{*}{ Monotropa hypopitys } & $\mathrm{BU}$ & Villarcayo & Ampliación corológica \\
\hline & $\mathrm{BI}$ & Zalla & Ampliación corológica \\
\hline & $\mathrm{VI}$ & Fontecha & Ampliación corológica \\
\hline Myosotis persoonii & $\mathrm{BU}$ & Mecerreyes & $3^{\text {a cita provincial }}$ \\
\hline \multirow[t]{2}{*}{ Narcissus gr. pseudonarcissus } & $S$ & $\begin{array}{l}\text { Castro Urdiales, Guriezo } \\
\text { y Liendo }\end{array}$ & Ampliación corológica \\
\hline & $\mathrm{BI}$ & Galdames y Güeñes & Ampliación corológica \\
\hline Neottia nidus-avis & BU & Santa María de la Cubilla & Ampliación corológica \\
\hline Nigritella gabasiana & BU & Espinosa de los Monteros & $\begin{array}{l}\text { Rebaja cota altitudinal } \\
\text { peninsular }\end{array}$ \\
\hline Omalotheca sylvatica & $\mathrm{BI}$ & Areatza & $\begin{array}{l}\text { Rebaja cota altitudinal } \\
\text { peninsular }\end{array}$ \\
\hline Onobrychis saxatilis & BU & Lerma & Ampliación corológica \\
\hline Ononis ramosissima & $\mathrm{BI}$ & Pozalagua & $\begin{array}{l}\text { Flora protegida / } \\
\text { Alejada franja litoral }\end{array}$ \\
\hline Ophrys lutea & $S$ & Sonabia & $\begin{array}{l}\text { Rebaja cota altitudinal } \\
\text { cántabra }\end{array}$ \\
\hline Ophrys speculum & $\mathrm{VI}$ & Markinez & Ampliación corológica \\
\hline Orchis coriophora & $\mathrm{BI}$ & Sondika & Ampliación corológica \\
\hline Orchis italica & BU & $\begin{array}{l}\text { Villacomparada de Rueda } \\
\text { y Extramiana }\end{array}$ & Ampliación corológica \\
\hline Ornithogalum bourgaeanum & BU & Villacomparada de Rueda & Ampliación corológica \\
\hline Ornithogalum pyrenaicum s. pyren. & $\mathrm{BU}$ & Villasuso de Mena & Ampliación corológica \\
\hline Orobanche hederae & $S$ & Castro Urdiales & $\begin{array}{l}\text { Franja costera oriental } \\
\text { cántabra }\end{array}$ \\
\hline Pedicularis schizocalyx & BU & Medina de Pomar & $\begin{array}{l}\text { Ampliación corológica } \\
\text { (nexo geográfico) }\end{array}$ \\
\hline Pentaglottis sempervirens & BU & Hozabejas & Ampliación corológica \\
\hline \multirow{2}{*}{ Pinguicula lusitanica } & $S$ & Castro Urdiales & Ampliación corológica \\
\hline & $\mathrm{BI}$ & Arcentales & Flora protegida \\
\hline
\end{tabular}




\begin{tabular}{|c|c|c|c|}
\hline Nombre científico & PR & Localidad & ¿Porqué se citan? \\
\hline Polygonatum verticillatum & $\mathrm{BU}$ & Espinosa de los Monteros & Ampliación corológica \\
\hline \multirow[b]{2}{*}{ Populus tremula } & $\mathrm{S}$ & Castro Urdiales & Cota altitudinal \\
\hline & $\mathrm{BI}$ & $\begin{array}{l}\text { Güeñes, Musques, Lemoiz, } \\
\text { Berango, Getxo, Trápaga } \\
\text { y Galdames }\end{array}$ & Cota altitudinal \\
\hline Primula elatior s. elatior & $S$ & Castro Urdiales & Cota altitudinal \\
\hline \multirow{2}{*}{ Primula veris s. columnae } & $\mathrm{Bl}$ & $\begin{array}{l}\text { Gorliz, Sopuerta } \\
\text { y Musques }\end{array}$ & $\begin{array}{l}\text { Franja costera vizcaína / } \\
\text { Ampliación corológica } \\
\text { (nexo geográfico) }\end{array}$ \\
\hline & $S$ & Castro Urdiales & $\begin{array}{l}\text { Franja costera oriental } \\
\text { cántabra }\end{array}$ \\
\hline Primula $x$ digenea & S & Castro Urdiales & $2^{\text {a }}$ cita provincial \\
\hline \multirow[t]{2}{*}{ Primula x polyantha } & $\mathrm{S}$ & Dobárganes & $3^{\text {a }}$ cita provincial \\
\hline & $B U$ & Villasuso de Mena & $1^{\text {a }}$ cita provincial \\
\hline Pulicaria odora & $\mathrm{Bl}$ & Zalla & Ampliación corológica \\
\hline \multirow[b]{2}{*}{ Pulsatilla rubra } & NA & Urbasa & $i 1^{\text {a }}$ cita provincial? \\
\hline & $\mathrm{BU}$ & $\begin{array}{l}\text { Mijangos, Quintanabaldo } \\
\text { y Brizuela }\end{array}$ & Flora protegida \\
\hline Ranunculus parviflorus & $\mathrm{VI}$ & Maturana & Ampliación corológica \\
\hline Rhamnus cathartica & BU & Villasuso de Mena & Ampliación corológica \\
\hline \multirow[t]{3}{*}{ Rhinanthus burnatii } & BU & Cernégula & Ampliación corológica \\
\hline & $\mathrm{Bl}$ & Leioa & Ampliación corológica \\
\hline & LO & Islallana & Ampliación corológica \\
\hline Rhus coriaria & $\mathrm{S}$ & Castro Urdiales & Especie alóctona \\
\hline Ribes petraeum & $\mathrm{BU}$ & Espinosa de los Monteros & $2^{\mathrm{a}}$ cita provincial \\
\hline Rorippa palustris & $\mathrm{Vl}$ & Elosu & $3^{a}$ cita provincial \\
\hline Rosa agrestis & $\mathrm{s}$ & Castro Urdiales & Cota altitudinal \\
\hline Rosa stylosa & S & Castro Urdiales & Ampliación corológica \\
\hline Ruta montana & BU & $\begin{array}{l}\text { Puerto de las Mazorras } \\
\text { y Santa Coloma }\end{array}$ & Ampliación corológica \\
\hline Salpichroa origanifolia & $\mathrm{Bl}$ & Getxo & $\begin{array}{l}\text { Especie alóctona / } \\
3^{\mathrm{a}} \text { cita provincial }\end{array}$ \\
\hline Saxifraga dichotoma & $B U$ & Villacomparada de Rueda & $\begin{array}{l}\text { Ampliación corológica } \\
\text { (nexo geográfico) }\end{array}$ \\
\hline Scrophularia peregrina & $S$ & Castro Urdiales & $1^{\text {a }}$ cita provincial \\
\hline Sedum sexangulare & $\mathrm{Bl}$ & Derio & $\begin{array}{l}1^{\text {a }} \text { cita margen derecha } \\
\text { vizcaína }\end{array}$ \\
\hline Senecio bayonnensis & $S$ & Castro Urdiales & $\begin{array}{l}\text { Franja costera oriental } \\
\text { cántabra }\end{array}$ \\
\hline
\end{tabular}




\begin{tabular}{|c|c|c|c|}
\hline Nombre científico & PR & Localidad & ¿Porqué se citan? \\
\hline Serapias lingua & $\mathrm{BI}$ & Barrika & Franja costera vizcaína \\
\hline Serapias parviflora & $\mathrm{BI}$ & Derio & Ampliación corológica \\
\hline Silene portensis s. portensis & $\mathrm{BU}$ & Mijangos & Ampliación corológica \\
\hline Silene scabriflora s. scabriflora & $\mathrm{BU}$ & Villacomparada de Rueda & Ampliación corológica \\
\hline \multirow{3}{*}{ Silybum marianum } & $\mathrm{BI}$ & Derio y Paules & Ampliación corológica \\
\hline & BU & $\begin{array}{l}\text { Alto de Ro, Covides y } \\
\text { Ordejón de Ordunte }\end{array}$ & $\begin{array}{l}\text { Ampliación corológica } \\
\text { (nexo geográfico) }\end{array}$ \\
\hline & $S$ & Valle & Ampliación corológica \\
\hline Sisymbrella aspera s. aspera & $\mathrm{BU}$ & Quintanilla del Coco & Ampliación corológica \\
\hline Sisymbrium irio & $\mathrm{BI}$ & Algorta & Ampliación corológica \\
\hline \multirow[t]{2}{*}{ Sisyrinchium angustifolium } & $S$ & Castro Urdiales & $\begin{array}{l}\text { Franja costera oriental } \\
\text { cántabra }\end{array}$ \\
\hline & $\mathrm{BI}$ & Areatza & Especie alóctona \\
\hline Sisyrinchium rosulatum & $\mathrm{BI}$ & Galdames y Bilbao & Especie alóctona \\
\hline Soliva sessilis & & & Correción \\
\hline Sorbus latifolia & $\mathrm{VI}$ & Urarte & Flora protegida \\
\hline \multirow{2}{*}{ Spergula arvensis } & $\mathrm{BI}$ & Bakio & Ampliación corológica \\
\hline & $\mathrm{BU}$ & Mecerreyes & Ampliación corológica \\
\hline \multirow[t]{2}{*}{ Spiranthes spiralis } & $\mathrm{BI}$ & Zamudio & Ampliación corológica \\
\hline & $\mathrm{BU}$ & Medina de Pomar & Ampliación corológica \\
\hline Stachys alpina & $\mathrm{BI}$ & Mañaria y Galdames & Ampliación corológica \\
\hline Taxus baccata & $\mathrm{S}$ & Castro Urdiales & Flora protegida \\
\hline Thalictrum minus s. minus & $\mathrm{BI}$ & Gorliz & Efecto pedinótico \\
\hline \multirow{3}{*}{ Tillia platyphyllos s. platyphyllos } & $\mathrm{BI}$ & Trucios & Ampliación corológica \\
\hline & $\mathrm{BU}$ & Villasuso de Mena & Ampliación corológica \\
\hline & $S$ & Castro Urdiales & $\begin{array}{l}\text { Cota altitudinal / } \\
\text { Franja costera oriental } \\
\text { cántabra }\end{array}$ \\
\hline Torilis nodosa & $\mathrm{BI}$ & Derio & Ampliación corológica \\
\hline \multirow{2}{*}{ Trifolium ochroleucon } & $\mathrm{BI}$ & Barrika y Ranero & $\begin{array}{l}\text { Cota altitudinal / } \\
\text { Franja costera vizcaína }\end{array}$ \\
\hline & $S$ & Guriezo & $\begin{array}{l}\text { Cota altitudinal / } \\
\text { Franja costera oriental } \\
\text { cántabra }\end{array}$ \\
\hline Ulmus glabra & S & Guriezo y Castro Urdiales & $\begin{array}{l}\text { Franja costera oriental } \\
\text { cántabra }\end{array}$ \\
\hline Urtica membranacea & $\mathrm{BI}$ & Laga & Franja costera vizcaína \\
\hline
\end{tabular}




\begin{tabular}{|c|c|c|c|}
\hline Nombre científico & PR & Localidad & ¿Porqué se citan? \\
\hline \multirow[t]{2}{*}{ Valeriana dioica } & $\mathrm{BI}$ & Getxo y Mungia & $\begin{array}{l}\text { Ampliación corológica } \\
\text { (nexo geográfico) }\end{array}$ \\
\hline & $S$ & Ampuero & Ampliación corológica \\
\hline Vandenboschia speciosa & $S$ & Castro Urdiales & Flora protegida \\
\hline Veronica verna & $\mathrm{BU}$ & $\begin{array}{l}\text { Quintanilla del Coco, } \\
\text { Mecerreyes y } \\
\text { Villacomparada de Rueda }\end{array}$ & $\begin{array}{l}2^{\mathrm{a}} \text { cita mitad septentrional } \\
\text { burgalesa }\end{array}$ \\
\hline Viburnum opulus & $\mathrm{BI}$ & Mungia & Franja costera vizcaína \\
\hline Vicia ervilia & $\mathrm{BU}$ & Espinosa de Cervera & $2^{\mathrm{a}}$ cita provincial \\
\hline Vicia loiseleurii & S & Navamuel & $\begin{array}{l}1^{\text {a }} \text { cita provincial / } \\
\text { Población finícola }\end{array}$ \\
\hline Viola alba & $\mathrm{BI}$ & Sopuerta & $4^{a}$ cita provincial \\
\hline Viola canina & $\mathrm{BU}$ & Nava de Ordunte & Cota altitudinal \\
\hline \multirow[t]{2}{*}{ Viola lactea } & $\mathrm{BI}$ & Getxo y Berango & Ampliación corológica \\
\hline & S & Castro Urdiales & $\begin{array}{l}\text { Franja costera oriental } \\
\text { cántabra }\end{array}$ \\
\hline Viola odorata & $\mathrm{BI}$ & Getxo & $\begin{array}{l}4^{a} \text { cita provincial / } \\
\text { Franja costera vizcaína }\end{array}$ \\
\hline \multirow{2}{*}{ Viola suavis } & $\mathrm{BI}$ & Getxo & Franja costera vizcaína \\
\hline & S & Santoña & Cota altitudinal \\
\hline \multirow{2}{*}{ Woodwardia radicans } & $\mathrm{BI}$ & Barrika & Flora protegida \\
\hline & $S$ & Castro Urdiales & Ampliación corológica \\
\hline
\end{tabular}

\section{Agradecimientos}

A nuestro buen amigo Daniel Gómez del IPE de Jaca por la ayuda en la identificación de la gramínea Festuca heterophylla y Agrostis hesperica. A Mikel Lorda por las claves de Crocus. Como siempre, a nuestros compañeros y amigos de Basozaleak (Sociedad micológica y botánica de Getxo) y acompañantes en las excursiones y paseos por la naturaleza, que anotamos en estricto orden alfabético, con la esperanza de que no hayamos cometido ningún olvido: Miguel Babace, Mángeles Bernaola, Marcos Casquero, Verónica Díaz, Eduardo Fidalgo, Manu Franco, Marisa Llorente, Javier Muñoz... responsables de los legit (y a menudo también de la identificación) de algunas de las citas de este artículo. 


\section{Bibliografía}

Aizpuru, I., Aseginolaza, C., Uribe-Echebarría, P.M., Urrutia, P., Zorrakin, I., 1999. Claves ilustradas de la flora del País Vasco y territorios limítrofes. Gobierno Vasco, Vitoria-Gasteiz.

Alejandre, J.A., García, J.M., Mateo, G., (eds.) 2006. Atlas de la flora vascular silvestre de Burgos. Junta de Castilla y León, Caja Rural de Burgos, Burgos.

Alejandre, J.A. (ed,..), 2008-2017. Adiciones y revisiones al Atlas de la flora vascular silvestre de Burgos I a X. Flora Montiberica.

Castroviejo, S. (coord.), 1986-2019. Flora Iberica. Plantas vasculares de la Península Ibérica e Islas Baleares. Real Jardín Botánico, CSIC, Madrid.

Durán, J.A., 2014. Catálogo de la flora vascular de Cantabria. Colección Monografías de Botánica Ibérica n. 13. Jolube Consultor y Editor Botánico, Jaca.

Durán, J.A., Blanco, E., Goñi, J., Valdeolivas, G., Varas, J., 2017. Adiciones y revisiones del Catálogo de la Flora Vascular de Cantabria, I. Flora Montiberica 64, 47-61.

Font Quer, P., 1993. Diccionario de Botánica. Ed. Labor, Barcelona.

Lorda, M., 2013. Catálogo Florístico de Navarra / Nafarroako Landare Katalogoa. Monografías de Bot. Ibérica 11.

कै०

La versión extendida de este trabajo se puede consultar en el link:

http://www.aranzadi.eus/fileadmin/docs/Munibe/mcn.2021.69.03_versionextendida.pdf

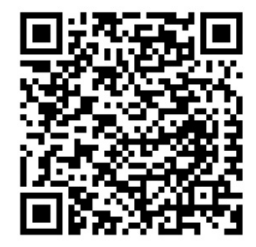

Fecha de recepción/ Date of reception: 16/06/2020

Fecha de aceptación / Date of acceptance: 12/01/2021

Editor Asociado / Associate editor: Cristina Herrero-Jaúregui 